\title{
Lectura y escritura de las representaciones sociales: hacia la conformación de una postura crítica en la educación superior
}

\author{
The reading and the writing of the social representations: \\ Toward a critical view in the university
}

Juliana Angélica Molina Ríos*

Recibido: 15 de marzo de 2012

Bibiana Yaneth Romero Chala*

Aceptado: 23 de mayo de 2012

\section{Resumen}

El presente artículo es producto de las investigaciones originadas en el ejercicio de la docencia en el campo de la escritura y de la lectura, las cuales son analizadas desde la teoría de las representaciones sociales. Se promueve la lectura crítica como un camino para mejorar las habilidades de lectura de los estudiantes. Además, la escritura se concibe como un escenario de acceso a la configuración de las representaciones. Para el abordaje de dichas rutas se presenta una reflexión inicial sobre el concepto de representación y su lectura crítica en los medios de comunicación. Se muestran algunas acciones de investigación realizadas en este campo y, posteriormente, se analiza el papel de la escritura como escenario de configuración de las representaciones sociales.

Palabras clave: representaciones sociales, lectura, escritura, educación superior, medios masivos.

\section{Abstract}

This paper is written as a result of carrying out research within university classrooms where reading and writing is taught in L1. Reading and writing are analyzed from Discourse Analysis, especially from the theory of social representations. Critical reading of the media as a way to improve reading skills in the students is encouraged. Furthermore, writing is promoted as means to construct social representations. To achieve this endeavor, an initial reflection on the concept of representation is presented. It is also showed how critical reading of the media helps identify social representations and how the role of writing becomes a tool for constructing social representations. To exemplify this process some research experiences based on a Discourse Analysis approach are displayed. Finally, the role of writing is analyzed as a scenario to construct social representations.

Keywords: Social representations, reading, writing, university, mass media.

* Pontificia Universidad Javeriana, Bogotá. Profesora de tiempo completo del Departamento de Lenguas, Facultad de Comunicación y Lenguaje. Magíster en Lingüística Española del Instituto Caro y Cuervo. Licenciada en Lingüística y Literatura con énfasis en Lingüística de la Universidad Distrital Francisco José de Caldas. Áreas de docencia e investigación: lingüística, lectoescritura, análisis del discurso, sociolingüística. Correo electrónico: juliana.molina@javeriana.edu.co

** Universidad de Medellín. Profesora de tiempo completo del Departamento de Ciencias Sociales y Humanas. Magíster en Lingüística Española del Instituto Caro y Cuervo. Licenciada en Lingüística y Literatura con énfasis en Etnoeducación de la Universidad Distrital Francisco José de Caldas. Áreas de docencia e investigación: lingüística, lectoescritura, comunicación organizacional, análisis del discurso. Miembro del grupo de investigación Lingüística y Comunicación Organizacional de la Universidad EAN. Correo electrónico: bibiromeroch@hotmail.com 


\section{Introducción}

La lectura y la escritura, sobre todo en la educación superior, se deben comprender como prácticas sociales articuladas a procesos de construcción, producción e interpretación de sentidos, pues por medio de ellas se genera conocimiento que transforma a los individuos y se crean comunidades en diversos ámbitos (cotidiano, académico, religioso, político, etc.). De allí el imperativo de pensar estas actividades más allá de su uso instrumental, puesto que ya no deben entenderse únicamente como herramientas de codificación y decodificación de signos lingüísticos.

En este sentido, Francisco González (citado en Pereira, 2009) aporta una afirmación que, en tono poético, ofrece con rigor una definición de la función esencial de la lectura como generadora de cultura:

La cultura, donde leer es primordial, tiene una etimología, que es cultivo, y eso nos une a la tierra, al esfuerzo, a la siembra y a la espera del tiempo apropiado y a las condiciones ambientales favorables para que germine la semilla. (p. 34)

Así, la lectura (y por ende, la escritura) como cultivo, implica construcción de identidades colectivas, de cosmovisiones, de transmisión y transformación de costumbres.

Desde esta perspectiva, la lectura y la escritura son vías para la configuración de representaciones sociales. Mediante de estas rutas se describe, califica, nombra e interpreta la realidad; además, son cauces para el flujo creativo de universos ficcionales que constituyen también representaciones de un orden especial. Las representaciones individuales y colectivas tienen lugar en el lenguaje, es ahí donde se reproducen, se validan o carecen de legitimidad. En consecuencia, se hallan articuladas a las prácticas lectoescriturales, de modo que son elaboraciones o reelaboraciones de los procesos de comunicación e interacción humanos.

El contexto anterior propicia el surgimiento de dos interrogantes fundamentales y atinentes a la educación superior: ¿Cuáles transformaciones sociales y culturales se producen o son posibles cuando se lee? ¿Qué papel desempeña la escritura en el acceso a la creación, reproducción y circulación de las representaciones sociales? En un mundo globalizado en el que la información tiende a desplazar el conocimiento porque suele confundirse con él; en una sociedad de consumo, digital, virtual y líquida (el concepto es de Bauman [2007]) donde la fugacidad prima en todo tiempo y lugar, y donde los medios masivos de comunicación inciden en las formas y estilos de vida "necesarios", la lectoescritura se configura como un producto cuyo uso es sinónimo de ser culto y letrado.

Frente a esta visión apocalíptica de dichas prácticas, el presente artículo reflexiona acerca de algunas experiencias de investigación originadas en los últimos años de docencia ${ }^{1}$ por medio de dos probables rutas de estudio en relación con las representaciones sociales (RS). La primera, relativa a la posibilidad

1 Como producto de esta experiencia hay dos artículos publicados y una reseña. El primero se titula: Molina, J y Jaramillo, C. (julio - diciembre, 2010). Las movilizaciones del 4 febrero y el 6 de marzo de 2008 en Bogotá, Colombia. Revista y Pensamiento, XXIX, 355-371. Bogotá - Cali: Facultad de Comunicación y Lenguaje - Pontificia Universidad Javeriana, Bogotá; Departamento de Comunicación y Lenguaje - Pontificia Universidad Javeriana, Cali. El segundo: Molina, J. (enero - junio, 2009). La representación social del fenómeno del desplazamiento forzado en la prensa colombiana. Revista Universitas Humanística, 38 (67), 128-146. Bogotá: Facultad de Ciencias Sociales - Pontificia Universidad Javeriana, y la reseña de Romero, B. "La representación social de los indígenas y de sus acciones (la defensa de sus territorios) en el diario El Tiempo". En: van Dijk, T. (2007). Racismo y Discurso en América Latina (pp. 214-220). Madrid: Gedisa. 
de proponer un ejercicio de lectura crítica de los medios que impacte el quehacer pedagógico de la enseñanza de la lengua. La segunda, atinente a la prácticas escriturales como instancias de acceso a la configuración de representaciones.

Para el emplazamiento de las rutas se presenta en principio una reflexión teórica acerca del concepto de representación social para entroncarlo con las prácticas lectoescriturales inherentes a los medios de comunicación. A continuación, se exponen los resultados de tres investigaciones adelantadas como parte de la labor docente, las cuales plantean la pertinencia de la lectura como que posibilidad de ejercer posturas críticas frente a dichos medios. Y posteriormente, se discute acerca de la escritura y su papel en la configuración de representaciones.

\section{Las representaciones sociales y los medios de comunicación}

La construcción de las representaciones sociales se ejerce en las interacciones simbólicas, lo que significa que su estudio debe localizarse en el lenguaje. En palabras de Irene Vasilachis, las representaciones son "construcciones simbólicas individuales y/o colectivas a las que los sujetos apelan o las que crean para interpretar el mundo, para reflexionar sobre su propia situación y la de los demás, y para determinar el alcance y la posibilidad de su acción histórica" (1998, p. 268). Con el lenguaje, al transportar significado de una persona a otra, se logra representar un objeto ausente, un suceso pasado o evocar el futuro.

Visto así, los medios de comunicación masiva cobran especial relevancia respecto de las representaciones y las prácticas de lectura y escritura. El lugar y el poder que estos ejercen en la sociedad se convierte en un componente cultural de las representaciones sociales:
[...] están en los medios de comunicación tanto en la mente de las personas y es necesario interceptarlas, ejemplificarlas y analizarlas en ambos lugares. Se trata de una teoría que confiere significación a los medios masivos de comunicación y a sus contenidos. Las representaciones no solo forman parte de la cognición sino también de la cultura. (Farr, 2003, p. 160)

Entonces las representaciones no solo tienen que ver con la descripción y categorización de la realidad, sino también con los valores socioculturales implicados en su elaboración.

Barrios, refuerza la anterior idea al afirmar que:

[...] las elaboraciones que realizan los medios de comunicación sobre la realidad tienen influencia en la audiencias [...]; utilizan una escala de valores que terminará constituyendo o reforzando el baremo sobre el cual se miden y entienden las acciones propias y ajenas y muestran determinadas concepciones del mundo que modelan las expectativas, creencias y opiniones de la audiencia. (2002, pp. 148-149)

De allí se infiere que es mediante la información recibida de los medios como clasificamos y establecemos juicios sobre los acontecimientos y también dirigimos nuestras acciones en la vida social, sin tener demasiado en cuenta los procesos de selección, filtrado, ocultamiento o sobrevaloración previos en la emisión de los mensajes.

En este sentido, los medios pueden producir patrones de comportamiento que generan expectativas individuales y sociales, las cuales moldean, en gran parte, a las sociedades.

Dado este panorama se encuentra que, por un lado, los productores de la información, al describir la realidad, la interpretan. Además, ellos están determinados, en mayor o menor grado, por sus propias 
limitaciones profesionales y personales. Profesionales porque el periodista actúa bajo ciertas condiciones institucionales impuestas (a veces no de manera explícita) por el medio de comunicación para el cual trabaja; y personales, porque depende de la amplitud o la restricción de sus experiencias de vida y conocimiento (a la vez permeado por su cosmovisión e ideología).

Por otra parte, y del lado del lector, no podemos olvidar que él está en capacidad de dudar de la veracidad de la información, y además de elaborar su propia interpretación, su posición crítica particular frente a la noticia, filtrada también por sus limitaciones, sus propias experiencias y creencias. Entonces está en condición de tratar de desentrañar por qué los medios dicen lo que dicen.

En el sentido propuesto, Luis Alfonso Ramírez (2001) asegura que:

[...] la imagen de una acción o de una persona, puede ser deformada y desprestigiada, pero también maquillada y realzada de acuerdo con las conveniencias, lo cual sucede con mucha frecuencia por los medios masivos de comunicación, especialmente. Lo interesante es que todos los problemas mencionados se ocasionan por el uso del lenguaje, ya sea por los procedimientos que se utilizan en la comunicación o por las formas de enunciación, es decir, por el procedimiento que se sigue en la combinación de las formas de expresión para montar el discurso. (p. 192)

De esta forma, los medios de comunicación son uno de los escenarios donde se representan, se reprimen o no se representan hechos de la sociedad, así como también se minimizan o maximizan, normalizan o anormalizan las acciones de nosotros y de los otros. Ellos son los que proponen qué y quién existe, y también cómo se debe nombrar. De suerte que, generalmente, la categorización de los actores se da en términos de: "buenos" vs. "malos", "nosotros" vs. "ellos", “yo" vs. "tú".
Asimismo, las agendas de medios definen cuáles problemáticas se deben tratar, ignorar o desarrollar: esta semana el tema de la salud, la próxima el de la seguridad ciudadana, la de más allá el de la corrupción, y así sucesivamente. Pareciera que la realidad representada cabe en secuencias semánticas prediseñadas para describir diversos y distintos sucesos. En suma, los medios de comunicación resultan categorizando la realidad de forma estandarizada, sin presentar nuevos formatos que logren interpelar al receptor.

En consecuencia, a menudo lo representado sustituye la misma realidad y, en este sentido, el lector puede aparecer como un lector perezoso (el concepto es de José A. León), o analfabeta funcional, pero también como un lector crítico. Desde este contexto, y tal y como lo señala Roncallo: "la prensa ha provocado cambios extraordinarios en las costumbres de lectura, así como en el carácter y la manera en que el hombre moderno percibe el mundo externo" (2006, p. 2). Precisamente las prácticas de lectura están inmersas en nuevas dinámicas sociales y en nuevas relaciones de poder, las cuales requieren lectores vivaces que se pregunten por el contenido mismo de la comunicación.

Al respecto, Sandra Araya (2002) resalta que en el estudio de la conformación de las representaciones sociales:

[...] las personas son concebidas como seres que piensan autónomamente y que producen y comunican constantemente representaciones y no como meras receptoras pasivas, por lo que cualquier determinismo social es rechazado. En la construcción de la realidad social el papel del Alter es significativo. Las personas se relacionan entre sí y en esta relación con los otros y las otras, elaboran observaciones, críticas, comentarios y "filosofías" no oficiales que tienen una influencia decisiva [...]. Por lo anterior, la teoría de las RS hace énfasis en la importancia de los procesos inferenciales presentes en la construcción de la realidad y en la insistencia 
de que la realidad es "relativa" al sistema de lectura que se le aplica. (p. 18)

Se observa, pues, que lejos de pensar en una dinámica unidireccional y determinista en la lectura de las representaciones que proponen los medios, se sostiene que no solo es plausible, sino también imperativo el ejercicio de lectores activos y propositivos frente a estos. Dicha dinámica puede propiciarse desde las educación superior, tanto en el ejercicio responsable e investigativo de los docentes (como que lectores de medios) como en la motivación de los estudiantes a dirigirse a estas prácticas del lenguaje desde la apropiación de una postura crítica.

En síntesis, el estudio de las representaciones en los medios de comunicación resulta pertinente en la educación superior no solo por la responsabilidad de formación disciplinar sino también por la reflexión crítica que el estudiante pueda desarrollar; así como el posible impacto social positivo que se pueda generar a partir de prácticas responsables de lectura y escritura.

\section{La lectura de las representaciones sociales}

A continuación se reseñan actividades de investigación que dan cuenta de cómo por medio de una lectura crítica de lo representado en los medios de comunicación, se puede cuestionar el determinismo vía única supuesto para los posibles lectores. Ello, tal como se planteó, es viable tanto en las experiencias investigativas de los docentes como desde la sensibilización de los estudiantes respecto de la necesidad de dirigir miradas más vigilantes sobre el conocimiento mediático.
Desde este panorama, la lectura se plantea como un ejercicio de pensamiento que permite que el estudiante se desarrolle como sujeto crítico. Dicho ejercicio involucra procesos de identificación, análisis e interpretación de temas, sujetos, acciones y, en un nivel más profundo, se logra desentrañar lo que no está explícito, se logra escuchar el silencio de un texto.

Así, se destacan los aportes de tres investigaciones relacionadas con la lectura crítica de la prensa y desarrollados desde la postura del análisis del discurso $(\mathrm{AD})$, en ellos se revisa el fenómeno de la representación social de los indígenas (Romero, 2005) del desplazamiento (Molina, 2007), y de las movilizaciones del 4 de febrero y el 6 de marzo del 2008 (Jaramillo y Molina, 2010) en Colombia.

El ejercicio de lectura de medios en estas investigaciones permitió evidenciar algunas particularidades acerca del modo como estos vehiculan y contribuyen a estabilizar las representaciones sobre hechos nucleares en la sociedad colombiana, y a su vez, contribuyó a la apertura de espacios de discusión y difusión académica.

Por ejemplo, en lo que respecta a la representación de las acciones y movilizaciones indígenas por la recuperación de los territorios ancestrales, ${ }^{2}$ las conclusiones revelan que el discurso mediante el cual se representa a los actores sociales en la prensa escrita, en este caso en El Tiempo, resulta ser selectivo, puesto que propone formas diferenciales de mostrar a los indígenas con respecto a otros actores como la policía y el gobierno.

Este manejo se evidenció en tres aspectos: el primero relativo a las formas de exclusión, por cuanto se inscribe a los indígenas como actores minimiza-

2 En esta investigación se revisa un corpus de once noticias presentadas en el diario El Tiempo durante el año 2003. 
dos o reducidos en su fuerza de acción social, y en el ocultamiento de las acciones negativas (asesinar, golpear e incumplir) de la policía y el gobierno. En segunda medida, en el modo de inclusión de los actores, el cual propone una diferenciación basada en la aparición de los indígenas como los ejecutores y responsables de las acciones negativas, mientras que la policía y el gobierno son representados como los agentes de acciones legítimas y deseadas.

Finalmente, en la estructuración de los diferentes contextos comunicativos en los cuales se presentan las acciones, se proponen actores como la policía y el gobierno como las autoridades para atribuir hechos a los indígenas y presentarlos de este modo como los agentes de lo negativo. Asimismo, en estos contextos las acciones sociales que no se ajustan al paradigma de lo establecido, cuando son ejecutadas por actores institucionales, son borradas o mitigadas. Lo anterior hace parte de una presentación parcial de la información y de una reproducción de la impunidad en los medios de comunicación.

Por su parte, en el análisis de la representación social del desplazamiento en la prensa nacional ${ }^{3}$ se adelantó un estudio de los modos de inclusión, exclusión, la representación de los actores, los titulares y los usos metafóricos que caracterizan dicho fenómeno. Tras el análisis, se señaló que la prensa escrita ha articulado una caracterización basada en la homogeneidad y los estereotipos frente a las víctimas del desplazamiento. Esta forma de situar en contexto es además fragmentada y superficial, puesto que los desplazados se presentan como objetos que se mueven en función de los intereses de otros actores (como los victimarios), quienes son minimizados en el discurso.
En conclusión, la asimetría expresa en el ocultamiento o sutil nombramiento que se hace de los responsables del desplazamiento forzado, frente a la sobreabundancia de referencias tipificadas de las víctimas, actúa como una forma simbólica de legitimización de las prácticas de injusticia social.

Por último, el estudio de las representaciones de las movilizaciones del 4 de febrero, "Un millón de voces contra la Farc", y del 6 de marzo de 2008, "Homenaje nacional a las víctimas del paramilitarismo", logró articular una lectura contrastiva de los diarios El Tiempo, El Colombiano y del semanario Voz. La mirada al discurso de la prensa situó, a partir del establecimiento de las microcategorías de los tópicos, tres temáticas centrales que fueron manejadas de modos sutilmente distintos por cada medio. Dichas categorías fueron: aspectos políticos, perspectiva de futuro y características de las marchas.

De acuerdo con el análisis de El Colombiano, en los aspectos políticos publicó la invitación a participar en la marcha del 4 de febrero, mientras que para la marcha del 6 de marzo inscribió el hecho como un antecedente determinante, pero matizó en la necesidad de no hacerse ilusiones al respecto. Además de una caracterización de las marchas, estableció la primera como el punto de referencia y medición para la segunda. A partir de la reflexión de la noción de conflicto armado y de los roles de los actores, se evidenció la ratificación de la asimetría moral tanto de las víctimas como de los victimarios.

En cuanto al semanario Voz, se identificó en la lectura de los tópicos una visión polarizada y politizada de la sociedad civil frente al conflicto, la cual se matiza con la caracterización de elementos negativos de la marcha del 4 de febrero y positivos de la marcha

3 En esta investigación se revisó un corpus conformado por cuarenta noticias emitidas en el año 2005 en los diarios El Tiempo, El Espectador, El Nuevo Siglo, El Heraldo, El Universal, El Colombiano, Vanguardia Liberal, El País, El Nuevo y Diario del Sur. 
del 6 marzo. Finalmente, El Tiempo evidenció una posición estrictamente política e invitó, mediante distintas estrategias argumentativas, a la participación en la marcha del 4 de febrero. Al igual que los otros diarios estableció esta marcha como referente para la otra y planteó un debate frente a la situación de los actores y la decisión de marchar a favor de unos o de otros, pero para la marcha del 6 marzo los inscribió a todos en el mismo nivel.

En síntesis, la investigación mostró que el cubrimiento mediático y la reacción política frente a las dos marchas fue distinto, tanto en el despliegue publicitario como frente a las posiciones políticas asumidas por los actores. En última instancia, el análisis de la representación condujo a observar la profunda polarización política que caracteriza al país, su relación con los agentes mediáticos y la pugna ideológica que vive la población civil.

Como puede apreciarse en esta investigación, la lectura, particularmente la de las representaciones sociales en el ámbito de la educación superior, ${ }^{4}$ es posible desde la consideración y desentrañamiento de la incidencia social que los medios de comunicación, como la prensa, tienen en la construcción de la realidad social. La fuerza con la cual estos se revelan en la estructuración de la cognición, hace necesarias las acciones de lectura responsables por parte de los educadores y aún más de aquellos que enseñan la lengua.

Ahora bien, la lectura atenta de estos fenómenos puede ser estimulada en los estudiantes, esto quiere decir que las actividades investigativas, las metodologías y los hallazgos deberían impactar seriamente las prácticas docentes. Es un hecho que nuestros estudiantes son lectores de medios: prensa, radio, televisión e internet constituyen universos transitados asiduamente en los cuales se intercambian y construyen los distintos sentidos que nutren la vida cotidiana.

En conclusión, se propone que la visión de lectura pasiva debe ser repensada para todos aquellos que participan del proceso educativo, pues tal como sostiene Araya:

La realidad social impone a su vez las condiciones de su interpretación por los sujetos, sin que ello implique un determinismo estricto. Esto significa que las matrices socioestructurales y los entramados materiales en los que están inmersas las personas definen su lectura de la realidad social, sus claves interpretativas y reinyectan en su visión de la realidad una serie de condicionantes que reflejan sus inserciones en la trama socioeconómica y en el tejido relacional. (2002, p. 19)

De ahí que la educación desempeñe un papel fundamental en la definición de los condicionantes que proporcionan las claves interpretativas de la lectura, por cuanto ella permitiría un espacio de ejercicio crítico y por ende, de negociación de los significados producidos por una cultura.

\section{Producción de la escritura: configuración de las representaciones sociales}

Pensar los procesos de escritura en la educación superior requiere de la reflexión frente al contexto social de producción de la escritura.

Se comprende que el contexto social en el cual se produce la escritura no se debe desvincular de las prácticas de ideología y poder en las cuales se produce un texto, es decir, no puede aislarse de la pregunta acerca de quién produce un texto, con qué fin y a quién lo dirige, puesto que a partir de dichos

4 Se sitúa este ámbito por cuanto las investigadoras se han desempeñado como docentes en la educación superior y han orientado la enseñanza de la lengua materna. 
interrogantes se pueden construir y reconstruir las representaciones sociales que emergen de los textos escritos.

La escritura, entendida como discurso, se constituye en un fenómeno político, cultural y social. Es una acción que representa sociedades, grupos, individuos, organizaciones, entre otros. Al respecto, van Dijk (2002) señala que "El discurso, de muchas maneras, construye, cambia, define y contribuye a las estructuras sociales". Así, cuando alguien hace uso de la escritura representa realidades normales o anormales, maximiza o minimiza ciertas acciones o actores con el objetivo de dar credibilidad a su discurso.

Lo anterior implica que un texto escrito no se considera como una sumatoria de palabras sino como un tejido que devela las interacciones que se generan en las prácticas sociales. El texto se presenta con su contexto, en el cual se develan las relaciones de poder, las ideologías, la dominación, el racismo y cualquier otra forma de interacción social. La escritura vista así permite que se reconozcan las voces, la polifonía que lleva consigo un texto, las categorías que representan, reprimen o no representan a las acciones o a los sujetos. En consecuencia, la escritura como práctica sociocultural exige que se conciba como una actividad recurrente y de constante cambio; implica que se supere el imaginario, aún presente, del texto como producto acabado. De ahí la importancia de la formación de los estudiantes en la educación superior para que logren no solo leer más allá de las líneas, sino también llegar a ser sujetos capaces de poner en juego todas las habilidades lingüísticas para lograr procesos de escritura con sentido incluyente y responsable. Es decir, se requiere trascender de la instrumentalidad del uso, pues dichos aspectos se deben articular a prácticas reales y significativas de escritura para los estudiantes y así lograr diálogos entre las disciplinas. Ello supone dejar a un lado la práctica de la escritura "petrificada" o "cementada" en la cual nada es modificable, para otorgarle otro papel a la misma y relacionarla con la posibilidad de ejercer el proceso mismo de la construcción de representaciones, es decir, vincularla con su papel productivo y creativo.

En otras palabras, la escritura se concibe como un proceso continuo que implica el razonamiento y la composición de lo que se quiere decir y del cómo se quiere enunciar. Según Carlino (2004), la escritura es un poderoso instrumento que permite representar, analizar, revisar y transformar el conocimiento. De hecho, múltiples investigaciones antropológicas, históricas, lingüísticas y psicológicas (Goody, 1996; Ong, 1987) otorgan a la escritura una significación relevante en el proceso de construcción de saberes. De suerte que esta no es un simple proceso de instrumentalidad de la comunicación, sino que tal como lo demuestra Carlino (2005) es una condición para la creación y la transformación de saberes, pues significa un ordenamiento particular de la conciencia y por ende, una forma singular de estructurar el conocimiento.

No obstante la importancia de la escritura es foco de múltiples discusiones en la educación superior, en esta etapa se evidencian los vacíos de los estudiantes a la hora de enfrentarse con la producción escrita. Como lo señala Carlino (2007), la reacción corriente de las instituciones o de los docentes de las disciplinas es atribuir dichas falencias a la formación académica que los estudiantes reciben en su etapa de educación básica, de hecho existe un imaginario donde se pretende que dichas falencias deben ser resueltas por el propio estudiante, dado el nivel de educación y de responsabilidad que asume al ingresar a la educación superior.

Sin lugar a dudas, esta mirada al proceso de la escritura hace más compleja la red de situaciones problemáticas para su producción, e incluso -a veces de maneras no conscientes- convierte a las instituciones 
y a sus currículos en centros de exclusión, pues antes que abrir los caminos y brindar las herramientas necesarias para que el estudiante domine los saberes disciplinares, ignora las dificultades o avoca a soluciones poco fructíferas -como cursos de lectoescritura en los primeros semestres- para una actividad $\tan$ compleja.

\section{Reflexión final}

A modo de reflexión final, se pueden pensar dos aspectos fundamentales:

- En cuanto a la lectura crítica de los medios que impacte el quehacer pedagógico, la educación superior y, en particular, los docentes tienen un rol protagónico en la formación de lectores críticos $\mathrm{y}$ vivaces que asuman la lectura no únicamente como un ejercicio de decodificación de signos, sino también como una práctica social mediante la cual se construyen representaciones sociales, se develan ideologías, poderes y la cultura en sí misma. Son los docentes los llamados a incentivar en los estudiantes procesos reflexivos desde y en la lectura.

- Y, frente a la producción de la escritura, se señala que esta requiere de una lectura crítica, como instancia de acceso a la configuración de representaciones sociales, mediante la cual se logre determinar cómo se construyen ciertos eventos sociales, es decir, identificar la configuración lingüística que determina en gran medida prácticas sociales y culturales.

En definitiva, la educación superior, aunque no es el único nivel de educación que interviene en la formación de lectores y escritores críticos, sí acarrea una función social en la construcción de una sociedad reflexiva y crítica.

\section{Referencias}

Araya, S. (2002). Las representaciones sociales, ejes teóricos para su discusión (Cuaderno 27 de ciencias sociales). Costa Rica: Flacso.

Barrios, O. (2002). Realidad y representación de la violencia. Salamanca: Universidad de Salamanca.

Bauman, S. (2007). Tiempos líquidos. Barcelona: Tusquets.

Carlino, P. (2004). El proceso de escritura académica: cuatro dificultades de la enseñanza universitaria. Educere, 8 (026), 321-327.

Carlino, P. (2007). Escribir, leer y aprender en la universidad. Buenos Aires: Fondo de Cultura Económica.

González, F., Moscovici, S. y Marková, I. (2003). La presentación de las representaciones sociales: diálogo con Serge Moscovici. En J. Castorina (Comp.), Representaciones Sociales. Problemas. teóricos y conocimientos infantiles (pp. 111152). Barcelona: Gedisa.

Goody, J. (1996). Cultura escrita en sociedades tradicionales. Barcelona: Gedisa.

Farr, R. (2003). De las representaciones colectivas a las representaciones sociales: ida y vuelta. En J. Castorina (Comp.). Representaciones Sociales. Problemas teóricos y conocimientos infantiles (pp. 153-175). Barcelona: Gedisa.

León, J. A . (1996). Prensa y educación. Un enfoque cognitivo. Buenos Aires: Aique.

Molina, J. (2009). La representación social del fenómeno del desplazamiento forzado en la prensa colombiana. Revista Universitas Humanística, 38 (67), 128-146. Bogotá: Facultad de Ciencias Sociales - Pontificia Universidad Javeriana. 
Molina, J y Jaramillo, C. (2010). Las movilizaciones del 4 febrero y el 6 de marzo de 2008 en Bogotá, Colombia. Revista y Pensamiento, XXIX, 355-371.

Ong, W. (1987). Oralidad y escritura, tecnologías de la palabra. México: Fondo de Cultura Económica.

Pereira, C. et ál. (2009). El papel de la lectura en la educación superior. Algunas reflexiones aplicadas en educación superior. Universitas tarraconensis: Revista de ciències de leducació, 217-235. Recuperado el 24 de octubre de 2011, de http://pedagogia.fcep.urv.cat/revistaut/ revistes/juny09/miscelania_article04.pdf.

Ramírez, L. (2001). Verdad y subjetividad en el discurso. En J, Bernal (Ed.), Lenguaje y Cognición (pp. 191-206). Bogotá: Instituto Caro y Cuervo -Universidad de Salamanca.

Rodríguez, T. (2003). El debate de las representaciones sociales en la psicología social. Relaciones, 93, 51-80.
Romero, B. (2007). La representación social de los indígenas y de sus acciones (la defensa de sus territorios) en el diario El Tiempo. En van Dijk, T. Racismo y Discurso en América Latina (pp. 214-220). Madrid: Gedisa.

Roncallo, S. (2006). El miedo hace el mensaje. La prensa escrita y el discurso del miedo: El Tiempo y El Colombiano. Bogotá: Centro de Competencia en Comunicación para América Latina. Recuperado de http://www.c3fes.net/docs/ c3seguridadcolombia.pdf

Vasilachis, I. (2003). Pobres, pobreza, identidad y representaciones sociales. Barcelona: Gedisa.

Van Dijk, T. (2002). El análisis crítico del discurso y el pensamiento social Revista Athenea Digital, 1, 6. Barcelona: Universidad Pompeu Fabra. 